\title{
CAUSAS DE EPILEPSIA TARDIA EM UMA CLÍNICA DE EPILEPSIA DO ESTADO DE SANTA CATARINA
}

\author{
MARCELO RIGATTI*, PAULO CESAR TREVISOL-BITTENCOURT**
}

\begin{abstract}
Resumo - Objetivo: Identificar em nossa sociedade as etiologias mais prováveis de epilepsia iniciada após os 18 anos de idade. Método: Estudo retrospectivo e descritivo na CME/SUS de Florianópolis de 1990 à 1998, analisando 120 prontuários de pacientes com epilepsia tardia, isto é, aqueles que iniciaram suas crises epilépticas dos 18 anos em diante. As variáveis coletadas foram: idade da primeira crise, etiologias prováveis, história mórbida familiar. Resultados: A prevalência encontrada para epilepsia tardia foi 29,48\%.Epilepsias parciais sintomáticas foram majoritárias e as etiologias mais frequentes e relevantes encontradas foram: cisticercose com cerca de $20 \%$, trauma crânio encefálico com 15\%, convulsão febril na infância com 5\% e 35\% da amostra foi classificada como idiopática. Conclusão: prevalência de epilepsia de início tardio em nossa sociedade é $29,48 \%$, cifra ligeiramente superior à relatada em países desenvolvidos (25\%). Muito provavelmente, esta diferença é consequência do caráter epidêmico de cisticercose entre nós. Além disso, trauma crânio encefálico e crises febris são etiologias comuns. Profilaxia de epilepsia é viável e urgentemente necessária em nosso Estado.
\end{abstract}

PALAVRAS-CHAVE: epilepsia, cisticercose, convulsão febril, trauma craniano.

\section{Etiologies of late-onset epilepsy in an epilepsy clinic of Santa Catarina- Southern Brazil}

ABSTRACT - Objective: To identify the most common etiologies of late-onset epilepsy in our society. Method: Retrospective and descriptive study in the CME/SUS of Florianópolis from 1990 to 1998, evaluating 120 handbooks of patients with late-onset epilepsy, that is, those that had initiated epileptic seizures of the 18 years in ahead. The collected variables were: age of the first seizure, etiologies and morbid familial history. Results: The prevalence rate found for late-onset epilepsy was $29.48 \%$. Most of the sample had partial symptomatic epilepsy and the most frequent and important were: cysticercosis with $20 \%$, head trauma with $15 \%$, febrile convulsions in infancy with $5 \%$ and $35 \%$ were classified as idiopatic. Conclusion: Prevalence of late-onset epilepsy in our society is $29.48 \%$, value slightly upper to the told one in developed countries (25\%). Very probably, this difference is consequence of the epidemic character of cysticercosis among us. Moreover, head trauma and febrile convulsions are common etiologies. Prophylaxis of epilepsy is viable and urgently necessary in our society.

KEY-WORDS: epilepsy, cysticercosis, febrile convulsion, head trauma.

Epilepsia é um distúrbio recorrente da bioeletrogênese cerebral. Ela é uma síndrome neurológica comum, tendo alta prevalência mundial, estimada entre 0,4 a $2 \%$ da população em geral $^{1,2}$. Países em desenvolvimento são os que aparentemente apresentam taxas mais elevadas de epilepsia, isto refletindo possivelmente uma maior exposição aos fatores de risco associados com epilepsia ${ }^{1}$. No Brasil, apesar da inexistência de estudos epidemiológicos adequados envolvendo diferentes regiões sócio-econômicas, supõe-se que algo em torno de 1 a $2 \%$ da população em geral esteja sendo acometida por algumas das formas de epilepsia ${ }^{2,3}$.

Universidade Federal de Santa Catarina (UFSC): *Acadêmico de Medicina da UFSC; **Professor de Neurologia da UFSC. Aceite: 1-junho-1999.

Dr. Paulo Cesar Trevisol Bittencourt - Disciplina de Neurologia - Hospital Universitário UFSC - 88040-970 Florianópolis SC - Brasil. Email: pcb@hu.ufsc.br 
Epilepsias poderão ser provocadas por praticamente qualquer condição que afete o córtex cerebral. Pode, por exemplo, ser consequência de anomalias congênitas, infecções, tumores, doenças vasculares, doenças degenerativas ou lesões traumáticas. Entretanto, em expressivo contingente destes pacientes nenhuma anormalidade será detectada com os métodos ora disponíveis. Por outro lado, em mais de 3/4 dos pacientes com epilepsia, as crises começam antes dos 18 anos. A razão para esta idade preferencial de início ainda não está totalmente esclarecida; porém, a vulnerabilidade do sistema nervoso em desenvolvimento à descarga exagerada é documentada tanto clínica como experimentalmente. Por esta razão, qualquer classificação das causas da epilepsia deveria portanto, antes de tudo, tentar distinguir entre as diferentes etiologias que acometem crianças e adultos ${ }^{4,5}$. Outro aspecto relevante é o aparente aumento da incidência de ataques epilépticos iniciando na vida adulta, observado na última década ${ }^{6}$. A despeito da sua alta prevalência, poucos são os artigos publicados em nosso meio sobre este tema, ignorando-se suas principais etiologias em nossa sociedade. Em consequência, a literatura estrangeira é habitualmente consultada e os dados oferecidos por estas publicações podem não refletir o encontrado em uma sociedade tão peculiar como a nossa ${ }^{1}$.

Por isso, decidimos levar a cabo este estudo pioneiro, com a expectativa de identificação das etiologias mais frequentes entre nós. Os dados obtidos estimularão uma política de combate as condições mais comumente associadas. Além disso, servirão de base para futuras comparações com estudos similares realizados em outras regiões do nosso país. Assim, o objetivo deste estudo é identificar as mais prováveis etiologias de epilepsia tardia em nosso meio.

\section{MÉTODO}

No presente estudo, retrospectivo e descritivo, foram considerados somente os pacientes com epilepsia avaliados na Clínica Multidisciplinar de Epilepsia da Policlínica Regional I (CME) SUS, do município de Florianópolis/SC, no período de 1990 a 1998. Esta clínica é a única no gênero do Estado e presta assistência a pacientes encaminhados das suas mais diversas regiões, possuindo facilidade de acesso a diversos recursos complementares, sejam de neuroimagem ou neurofisiológicos. Ela iniciou suas atividades em 1990, e o seu núcleo é composto por um médico neurologista, uma psicóloga, um assistente social, uma estudante de enfermagem da UFSC e dois acadêmicos de medicina da mesma universidade. Este grupo é complementado por um secretário geral, responsável pela recepção de pacientes e familiares, bem como pela identificação inicial dos mesmos e também pela orientação ao final do atendimento e agendamento das novas reavaliações.

Foram considerados epilépticos todos aqueles cujo diagnóstico foi estabelecido após avaliação pela equipe multidisciplinar da CME/SUS, liderada por neurologista com especialização em epileptologia. Pacientes com crises epilépticas únicas ou aqueles com crises não genuinamente epilépticas, ou ainda sem definição diagnóstica, foram desconsiderados para os fins deste estudo.

Nesta pesquisa as seguintes variáveis foram consideradas: idade da primeira crise, etiologias prováveis; história mórbida familiar positiva para epilepsia; entendendo-se aqui que, toda vez que, o paciente ou acompanhantes informavam convictamente sobre a existência ou não de parentes consanguíneos com epilepsia, a história era considerada respectivamente positiva ou negativa; caso pairassem dúvidas ou não soubessem, este item era ignorado.

Os procedimentos utilizados para a inferição diagnóstica das possíveis causas implicadas na epileptogênese foram os seguintes: epidemiologia consistente com a possibilidade de teníase no passado pelo paciente ou familiares próximos e presença de lesões típicas de neurocisticercose (NC) em estudos tomográficos; então a causa provável considerada era NC. Quando obtinha-se a informação da ocorrência de crises epilépticas associadas com febre, entre $0-5$ anos de idade, convulsão febril era aventada como hipótese etiológica mais provável, lembrando que em Santa Catarina crises febris são comumente denominadas pela população leiga como ataque de vermes ou ataque de bicha. Agora, caso houvesse ocorrido evidência de hipóxia perinatal ou uso de forceps, enfim acidentes obstétricos relevantes de diversos tipos, na ausência de outros fatores predisponentes, esta possibilidade era aventada como a mais provável. Aqueles que referiam no passado meningite ou outras infecções do sistema nervoso central (SNC), ou evidenciavam tumor cerebral, ou que já tinham sido afetados por infartos cerebrais, ou tinham malformação arteriovenosa, foram enquadrados dentro das respectivas prováveis origens: meningite/ outras infecções; neoplasia; doença vascular cerebral; malformação arteriovenosa. Caso o início da epilepsia estivesse cronologicamente relacionada com traumatismo de crânio, não importando sua magnitude, então a causa atribuída seria trauma crânio encefálico (TCE). 
Todo aquele paciente cuja história clínica, exame neurológico e exames complementares sugerissem mais de uma possibilidade etiológica, eram enquadrados como de etiologia multifatorial.

Uma seleção aleatória da amostra foi implementada, com revisão de todos os prontuários. Aqueles identificados como portadores de epilepsia de início tardio foram separados para esta investigação.

Por não existirem estudos prévios que demarquem quando ou com que idade a epilepsia deveria ser chamada de tardia, resolvemos adotar o marco 18 anos, ou seja, todos os pacientes que debutaram epilepsia com idade igual ou superior a 18 anos.

\section{RESULTADOS}

Na CME foram pesquisados 407 prontuários, de pacientes acompanhados por epilepsias diversas, deste total foram separados aqueles que possuíam idade igual ou superior a 18 anos quando ocorreu a primeira crise, resultando em uma amostra de 120 pacientes $(29,48 \%)$, sendo que os restantes $(70,51 \%)$ apresentavam epilepsia desde a infância ou puberdade.

Quanto às prováveis causas para estes 120 pacientes, foram detectadas 10 condições etiológicas distintas. Na Figura 1, cada esfera com cores distintas entre elas corresponde a uma causa diferente, exemplificando, a esfera maior e central de cor marron escura na parte superior da figura representa a NC, já as quatro outras menores que a interceptam e de cores azul claro, verde claro, amarelo claro e marron claro são respectivamente: TCE, convulsão febril, idiopáticas e malformação arteriovenosa. As esferas da base da Figura I de cores roxa, vermelho, verde escuro, cinza, azul escuro representam respectivamente: transtornos no parto, neoplasia, meningite, doença vascular cerebral e criptogênicas. Porém, quando duas esferas se interceptam, existe para " $x$ " pacientes duas etiologias possíveis. Por exemplo, $19,16 \%$ dos pacientes tinham somente como provável etiologia NC e $15 \%$ somente TCE, porém, 3,33\% tinham as duas como possíveis etiologias, sendo impossível distinguir com segurança qual delas foi a desencadeadora do sintoma.

$\mathrm{Na}$ investigação se havia alguma história familiar, a positividade foi de $18 \%$, ou seja, 22 pacientes afirmaram que havia alguém na família com história de epilepsia. Para $22 \%$ dos pacientes a história era negativa e em $60 \%$ das vezes a informação não era consistente.

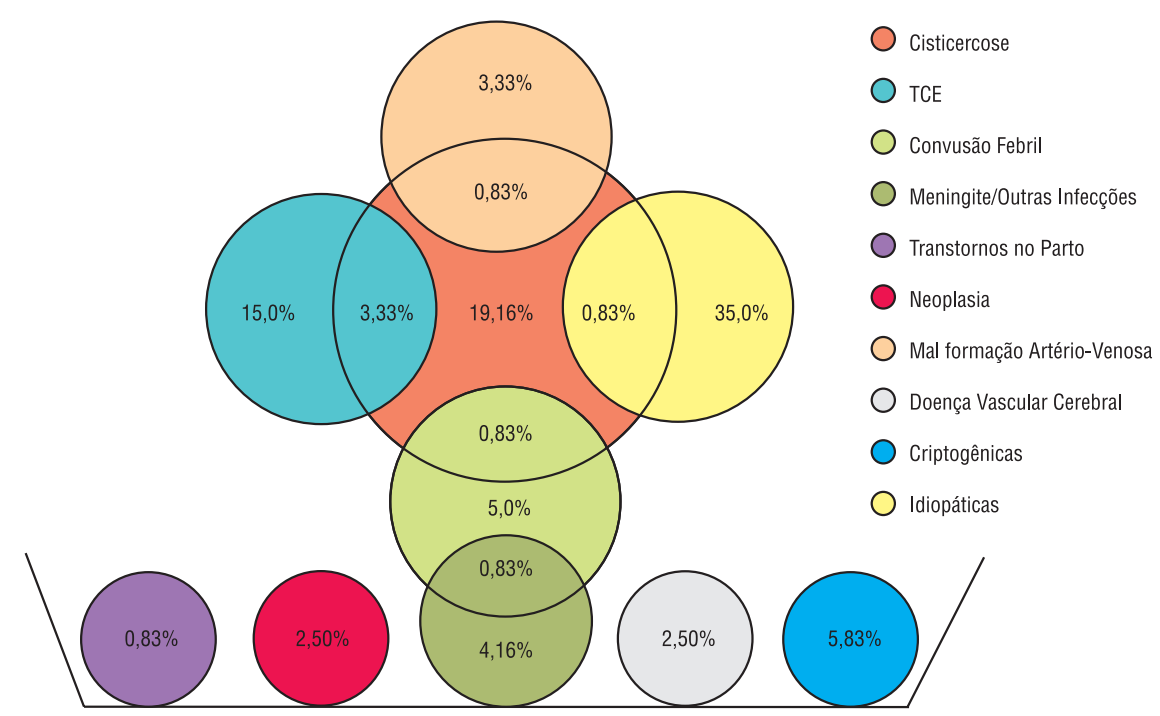

Figura 1. Etiologias de epilepsia tardia na CME/SUS. 


\section{DISCUSSÃO}

Epilepsia é condição com expressiva prevalência afetando entre $0,4-2 \%$ da população mundial ${ }^{2,7}$. Nos países desenvolvidos aceita-se que aproximadamente $25 \%$ dos pacientes iniciarão suas crises após os 18 anos de idade ${ }^{8}$. Cifras similares foram observadas em nosso estudo, pois dos 407 pacientes investigados, a taxa de epilepsia tardia encontrada foi $29,48 \%$ e mais de $3 / 5$ iniciaram suas crises antes dos 18 anos. Porém, notamos discreta elevação do índice de epilepsia tardia entre nós, quase $5 \%$. Se levarmos em consideração as principais etiologias, poderíamos justificar a diferença obtida, já que as etiologias mais prováveis, nas nações civilizadas, seriam: lesões vasculares, TCE, intoxicações por álcool e drogas, neoplasias, infecções e idiopáticas ${ }^{4,5}$. Em nossa investigação, além das citadas, encontramos como importantes etiologias: cisticercose e convulsão febril. É provável que peculiaridades do nosso Estado sejam a melhor explicação para esta sutil diferença. Nosso estágio de desenvolvimento nos coloca entre extremos, como por exemplo, Canadá - Uganda; por isso, temos etiologias comuns a estas distintas sociedades. Exemplificando, possuímos prevalência de NC, típica de terceiro mundo ${ }^{1,9}$, combinada com taxas de TCE que excedem em muito ao verificado nos países desenvolvidos ${ }^{10,11}$. Aliás, apesar da nossa fama de produzir bons esportistas (e pilotos também), somos pessimamente mal educados no trânsito. Neste aspecto parece que espelhamos o Brasil, que entre mortos e feridos, ainda continua produzindo a estúpida soma de uma guerra do Vietnã ao ano. Dessa maneira encontramos altos índices de TCE como causa de epilepsia tardia entre nós, responsável por cerca de $15 \%$ da amostra. Tal cifra atesta a nossa profunda falta de interesse em técnicas de prevenção dos acidentes de trânsito e de como minimizar seus efeitos nos envolvidos. Nós julgamos que este fato, per se, deveria ser merecedor de maior atenção por parte das autoridades da área, assim como despertar a classe empresarial e a sociedade em geral para a realização de campanhas eficientes que visem a um real controle deste problema endêmico.

Por outro lado, foi notável perceber que pelo menos $5 \%$ da amostra tinha ataques febris na infância como a mais provável etiologia da epilepsia de início tardio. Este elevado percentual entre nós, remete-nos a algumas indagações; será que nossa população infantil é vítima de mais doenças infecciosas que as de outras sociedades? Ou será que métodos para sua pesquisa são falhos? $\mathrm{Na}$ cultura açoriana, predominante entre nós, crises febris são definidas pela população leiga como ataque de vermes ou ataque de bicha. É bastante provável que cada sociedade tenha expressões bem típicas, como as citadas, para denominar o que alguém já definiu como confusão febril, tal a gama de dúvidas que envolvem esta entidade. Assim, nós acreditamos que esta possibilidade etiológica está artificialmente diminuída por histórias clínicas deficientes nas mais diversas sociedades, inclusive naquelas desenvolvidas. $\mathrm{O}$ fato é que, crises febris podem não ser tão benignas como afirmam alguns autores ${ }^{12-14}$ e sua capacidade em provocar esclerose mesial temporal e hipocampal está fartamente documentada atualmente ${ }^{2,15}$. Para reforçar nossa argumentação, gostaríamos de realçar que, em nossa amostra, todos os pacientes identificados com esta etiologia exibiam típica esclerose em estudos com ressonância magnética, sugerindo ser esta realmente, a mais provável desencadeadora de epilepsia nestes pacientes. E desde que esta é uma das causas com boa responsividade cirúrgica, sua identificação precisa é imperativa nos dias atuais.

Doenças vasculares cerebrais, uma frequente etiologia em países desenvolvidos ${ }^{8,11,15,16}$ não ocuparam lugar tão importante quanto o observado naquelas nações. No presente estudo, encontramos a ínfima taxa de 2,5\% naqueles pacientes com epilepsia iniciada tardiamente. Talvez a melhor explicação para este achado seja o fato de que naquelas sociedades a expectativa de vida seja maior e as doenças vasculares típicas da senilidade serem obviamente mais frequentes. Contudo, vivenciamos um real paradoxo tropical: apesar de crônico desgoverno, nossa população idosa encontra-se em rápida progressão de modo que epilepsia tardia, relacionada a esta etiologia, deverá sofrer expressivo incremento nas próximas décadas.

Já quando investigamos neoplasia como etiologia ${ }^{5,17}$, encontramos a taxa de 2,5\%, mostrando pouca diferença quando comparamos com países tecnologicamente mais avançados ${ }^{16,18}$. Isto pode 
significar que neoplasia cerebral tem incidência similar em qualquer parte do mundo, independendo de qualquer classificação sócio-econômica e cultural prévia.

Porém, em uma proporção substancial dos pacientes, a etiologia persistiu incognitamente multifatorial. Quando nos deparamos com duas possibilidades simultâneas, como por exemplo NC e TCE, distúrbios muito prevalentes em nosso meio, foi impossível para determinarmos qual deles era o responsável pela lesão epileptogênica. Para tal necessitaríamos de tecnologia sofisticada ainda não disponível em nosso serviço. E mesmo em sociedades avançadas onde estes recursos são disponíveis, alguns casos serão de difícil resolução. É possível que limitações desta natureza tenham contribuido para que num contingente expressivo da nossa amostra a origem tenha permanecido obscura. Entretanto, nós acreditamos que avanços nas técnicas diagnósticas, assim como sua disponibilidade em nossa sociedade, contribuirão no futuro para identificar melhor duas das principais causas de epilepsia na população em geral. A primeira delas, é a suscetibilidade herdada geneticamente, a segunda é a infecção sub-aguda ou crônica sem sintomas clínicos exuberantes ${ }^{4,17}$. Considerando hereditariedade, notamos que pacientes com parentes consanguíneos com epilepsia foram 18,33\%. Embora seja tentador atribuir responsabilidade à predisposição genética, deve-se lembrar da alta prevalência de NC em nosso meio, fato que influencia decisivamente na interpretação da positividade familiar, ou seja, o paciente tem história familial positiva para epilepsia idiopática ou sintomática? Nestes casos não estaria $\mathrm{NC}$ afetando vários membros de uma mesma família? Aliás, enfocando NC, sabe-se que em muitos países subdesenvolvidos ela é extremamente comum, chegando ao acometimento de $5 \%$ da população em geral. Já em países mais desenvolvidos, isto fica na ordem de 0,3 a $0,9 \%$. Ainda que a relação causal entre cisticercose e epilepsia não tenha sido ainda bem esclarecida, crises epilépticas são os sintomas mais comuns de $\mathrm{NC}^{1,19,20}$. Na nossa amostra, cisticercose ocupou lugar de atenção e destaque, sendo identificada como a responsável em aproximadamente $20 \%$ dos epilépticos tardios. Este dado isolado sugere que não estamos tão desenvolvidos quanto aparentamos; pois, se extrapolarmos para o total da população epiléptica em questão, chegaremos aos $7 \%$, conferindo com a literatura africana.

A incidência vergonhosamente elevada de NC entre nós, como causa potencial de epilepsia, deveria ser encarada como um atestado de incompetência por parte daqueles responsáveis pela administração da saúde pública em nosso estado. É tragi-cômico constatar que apesar de nós nos infectarmos com a mesma facilidade de africanos e asiáticos, nós nos diagnosticamos com a acurácia de escandinavos. Foi melancólico constatar que em nosso Estado, a Europa brasileira, NC parece ser a causa maior de epilepsia de início tardio. Mais constrangedor é perceber que esta é uma causa perfeitamente erradicável, bastando tão somente o desejo político de combatê-la; senão por altruísmo, pelo enorme dispêndio financeiro gasto no tratamento destes pacientes e também pela manutenção do nosso status de grande produtor de comida sadia ${ }^{1}$.

Finalmente, desde que nosso estudo identificou NC, TCE e crises febris como as principais etiologias de epilepsia tardia; como todas elas são condições passíveis de controle, nós podemos inferir que no futuro, muito provavelmente, teremos uma prevalência de epilepsia similar àquela observada atualmente nos países desenvolvidos ${ }^{2}$.

Agradecimentos - A valorosa colaboração do Dr. Antônio Carlos Marasciolo é reconhecida. Cristini Piacentini Boppre e Dangle Rigatti foram pessoas fundamentais na obtenção dos dados.

\section{REFERÊNCIAS}

1. Trevisol-Bittencourt PC, Figueiredo R, Silva NC. Neurocisticercose em pacientes internados por epilepsia no Hospital Regional de Chapecó- região oeste do Estado de Santa Catarina. Arq Neuropsiquiatr 1998;56(Supl.1):53-58.

2. Trevisol-Bittencourt PC. Redução de drogas em pacientes com epilepsias refratárias a politerapia anti-epiléptica. Dissertação de Mestrado, Universidade Federal de Santa Catarina, Florianópolis, 1993.

3. Trevisol-Bittencourt PC. "Brasilepsia”. JLBE 1992;5:31-32.

4. Gonzalez JLG, Martinez MP. Etiology of epileptic crisis in the geriatric patient results of a retrospective study. Arch Neurobiol 1991;54(Suppl. 4):135-139. 
5. Annegers JF, Rocca WA, Hauser WA. Causes of epilepsy: contributions of the Rochester epidemiology project. Mayo Clin Proc 1996;71:570-575.

6. Alberca R, Moreno A. Late onset epilepsy. Neurology 1996;11(Suppl 4):72-79.

7. Hauser WA, Annegers JF, Kurland LT. Incidence of epilepsy and unprovoked seizures in Rochester, Minnesota. Epilepsia 1993;34:453-468.

8. Alcon A. Etiological and prognostic factors in the late onset epilepsy. Neurology 1995;23(Suppl.120):285-289.

9. Garcia HH, Gilman RH, Tsang VC, Gonzalez AE. Clinical significance of neurocysticercosis in endemic villages. The cysticercosis working group in Peru. Trans R Soc Trop Med Hyg 1997;91(Suppl 2):176-178.

10. Ivan LP. The impact of head trauma on society. Can J Neurol Sci 1984;11:417-420.

11. Ryglewicz D. Epilepsy after stroke. Neurol Neurochir Pol 1992;26(Suppl.1):83-89.

12. Engel J. Update on surgical treatment of the epilepsies. Neurology 1993;43:1612-1617.

13. Wyllie E. Cortical resection for children with epilepsy. AJDC 1991;145:314-319.

14. Gram L. Epileptic seizures and syndromes. Lancet 1990;336:161-163.

15. Munõz MVR, Li LM, Bittencourt PCT, et al. The prevalence and social characteristics of epilepsy in a small azorean community in southern Brazil. Abstracts VIII Congresso Panamericano de Neurologia, Montevideo, 1991.

16. Chow SY, Hsi MS, Tang IM, Fong UH. Epilepsy and intracranial meningiomas. Chung Hua I Hsueh Tsa Chin (Taipei) 1995;55(Suppl.2):578-1337.

17. Smith MH. Late-onset epilepsy. Br J Clin Pract 1994;48(Suppl 2):87-90.

18. Fenelon G, Gray F, Moulignier A, Mikol J, Pialoux G Thiebaut JB. AIDS - associated progressive multifocal leukoencephalopathy revealed by new-onset seizures. Am J Med 1995;99(Suppl.1):64-68.

19. Andriantsimahavandy A, Lesbordes JL, Rasoaharimalala B, et al. Neurocysticercosis: a major aetiological factor of lateonset epilepsy in Madagascar. Trop Med Int Health 1997;2(Suppl.8):741-746.

20. Carpio A, Escobar A, Hauser AW. Cysticercosis and epilepsy : a critical review. Epilepsia 1998;39(Suppl 10):1025-1040. 